\title{
Narrative review: recent advances in doll therapy for Alzheimer's disease
}

\author{
Xiaoyan Cai, Lu Zhou, Peiying Han, Xiaolan Deng, Huanhuan Zhu, Fang Fang, Zhi Zhang \\ Department of Geriatrics, Nanjing Drum Tower Hospital, the Affiliated Hospital of Nanjing University Medical School, Nanjing, China \\ Contributions: (I) Conception and design: X Cai, F Fang; (II) Administrative support: X Deng; (III) Provision of study materials or patients: L Zhou, \\ H Zhu; (IV) Collection and assembly of data: P Han, X Cai; (V) Data analysis and interpretation: X Cai, F Fang, Z Zhang; (VI) Manuscript writing: \\ All authors; (VII) Final approval of manuscript: All authors. \\ Correspondence to: Zhi Zhang. Department of Geriatrics, Nanjing Drum Tower Hospital, The Affiliated Hospital of Nanjing University Medical \\ School, Nanjing, China. Email: 53635388@qq.com.
}

\begin{abstract}
Alzheimer's disease (AD) is a severe neurodegenerative disease which impacts the quality of life in elderly patients and imposes a significant burden to families and caregivers. The prolonged life expectancy and rapidly increasing world population significantly increased the morbidity. Although it has been widely reported that the aggregation of Beta amyloid and neurofilaments is the most significant pathological change. Currently, there is no effective drug therapy for $\mathrm{AD}$, and the potential risks of pharmacotherapy remain concerns. This article reviews the recent research on Doll therapy (DT), a widely used non-drug therapy on $\mathrm{AD}$ patients, especially its clinical effectiveness and precautions in treating $\mathrm{AD}$, with an attempt to further alleviate the mental symptoms and improve the health status of $\mathrm{AD}$ patients. Thus, nonpharmacological treatments of $\mathrm{AD}$ have become an area of intense research interest in recent years. DT is a person-centered therapy that can improve both the mental and cognitive status and the quality of life in AD patients. Although there remains ethical controversy about the DT on AD patients, its positive effect has been proved. Moreover, a standards manual is required to stipulate the range of application, the time course for treatment and withdraw the toy from the patients.
\end{abstract}

Keywords: Doll therapy (DT); Alzheimer's disease (AD); advance; review

Submitted Jan 27, 2021. Accepted for publication Apr 24, 2021.

doi: 10.21037/apm-21-853

View this article at: http://dx.doi.org/10.21037/apm-21-853

\section{Overview}

Alzheimer's disease (AD) is a progressive neurodegenerative disease. It is mainly featured by memory impairment but is often accompanied by symptoms such as language impairment, emotional instability, and decreased mobility. According to the statistics released by Alzheimer's Disease International (ADI) in 2018 (1), 50 million people are living with dementia globally, with AD contributing to approximately $60-70 \%$ of these cases (2-5). Without effective treatment and response measures, the number of people with dementia is expected to increase to 82 million in 2030 and 152 million in 2050. There are currently no effective treatments that can stop or reverse $\mathrm{AD}$, and the effectiveness and adverse effects of pharmacological treatments remain unsatisfactory $(3,6,7)$. Some nondrug therapies, such as reminiscence therapy, music therapy, aromatherapy, and doll therapy (DT), have been shown to be effective in temporarily relieving or improving symptoms and enhancing the quality of life. Among them, DT has been widely investigated due to its simplicity and costeffectiveness (8-11). Here, we review the studies related to the treatment of $\mathrm{AD}$ with $\mathrm{DT}$, with an attempt to better understand the effectiveness and clinical applications of this nondrug therapy.

We present the following article in accordance with the Narrative Review reporting checklist (available at http:// dx.doi.org/10.21037/apm-21-853). 


\section{Definition}

There is no uniform definition for DT. According to Pezzati et al. $(10,12,13)$, DT is a nonpharmacological intervention aimed at reducing behavioral and psychological disorders in institutionalized patients with dementia. It is effective in promoting and maintaining the affectiverelational dimension of attachment caregiving and the attentive dimension of exploration in patients with advanced stage of dementia, thus promoting clinically significant improvements in the ability to relate with the surrounding world. This therapy has been adopted and applied in longterm care facilities as a care tool to address the cognitive, behavioral, and physical problems of people living with dementia $(9,14)$.

\section{Theoretical rationale}

The theoretical rationale of DT is based on the concept of attachment theorized by Bowlby in 1980, with selfprotective mechanisms functioning as its biological basis. While most of the early studies on the attachment theory were focused on infants, in 1987, Hazan et al. (15) explored the possibility that romantic love in adults is an attachment process. As an important part of healthy development, attachment contributes to the ability of human beings to survive and adapt better to their environment and society. People have a need for attachment, and they have a tendency to seek and maintain proximity to a specific person when under stress. Miesen (16) has proposed the use of attachment theory as the key to the behavior of patients with dementia and describes the experience of a person with dementia as " a battle against powerlessness, disruption of daily existence and emotional collapse, similar to the basic reaction of anyone after a trauma of any nature or impact". Therefore, for AD patients, attachment is often considered to be an essential psychological need due to the physical and psychological impairment caused by the disease (17).

\section{Classification}

Dolls used for AD patients include both empathy dolls and anatomically correct dolls. Empathy dolls are typically ordinary toys, and anatomically correct dolls are more realistic. No study has compared the clinical effectiveness of these two doll types.

\section{Clinical values of DT for AD}

\section{Improving cognitive function and memory}

The core symptom of $\mathrm{AD}$ is the progressive impairment of language and cognitive function, and DT helps to improve cognitive functions (such as memory and language). Cai et al. performed a controlled trial in $186 \mathrm{AD}$ patients and found 93 patients in the DT group showed significantly improved language, place judgment, memory, movement, and cognitive function compared to 93 patients in the control group (treated with conventional therapy). Balzotti et al. (11) applied DT in $10 \mathrm{AD}$ patients, and the DT group showed significant improvements in agitation, irritability, and other psychological symptoms relative to controls; the patients' interaction and familiarity with the dolls facilitated the formation of communication and attachment relationships, reduced behavioral and emotional disturbances, delayed the progression of the disease to a certain extent, and improved the cognitive function of patients.

\section{Reducing unsafe behaviors and psychotropic drug use}

Several studies have shown that DT can reduce the use of antipsychotic drugs and decrease unsafe behaviors in AD patients. Juh et al. (18) conducted a controlled study on $72 \mathrm{AD}$ patients: 22 patients in the control group received conventional treatment and 52 patients in the DT group were given DT plus conventional treatment. The results showed that the Agitated Behavior Scale (ABS) score was significantly lower in the DT group than in the control group, indicating that DT was beneficial in relieving the agitated behavior of AD patients. Meanwhile, Green et al. $(8,10)$ found that DT was associated with less haloperidol use and fewer manic and agitated behaviors.

\section{Improving mood and mental well-being}

Most $\mathrm{AD}$ patients have a variety of mood-related symptoms, including anxiety, depression, panic disorder, and mania. Burke et al. (19) reported an increased incidence of depression of up to $69 \%$ in $712 \mathrm{AD}$ patients. $\mathrm{AD}$ patients are more likely to develop loneliness, despair, depression, and other negative emotions due to long-term social isolation, and DT can meet their emotional needs (e.g., 
attachment and respect), alleviate their negative emotions, and enhance their feeling of well-being. In a study performed by Moyle et al. (14), 3-week intervention with DT provided older people living with dementia with both enjoyment and emotional comfort/calm.

\section{Improving the quality of life and health status}

Patients with advanced $\mathrm{AD}$ can no longer live independently and require long-term care, which impacts the entire family, society, and the health care system. McCready et al. (9) performed a controlled trial in $87 \mathrm{AD}$ patients and found each dimension of quality of life was improved in 42 patients in the DT group compared with the 45 patients in the control group (treated with conventional therapy). Mitchell et al. (20) observed that $\mathrm{AD}$ patients who received DT had increased dietary intake and higher awareness of food, while Green et al. (8) concluded that DT was beneficial in improving sleep quality and the cooperation of patients.

\section{Precautions for DT}

An approval must be obtained from the medical ethics committee of a hospital before the use of DT in an AD patient. Before the use of dolls, the participants and their families should be informed of the purpose of the treatment orally or in writing, and a written informed consent form must be signed. Appropriate dolls should be selected and dressed with baby clothes. The ownership of a specific doll should not be confused when 2 or more $\mathrm{AD}$ patients receive DT simultaneously.

DT should be suspended immediately if the patient shows any pain or discomfort. The patient's attitude must be closely monitored during the introduction of a doll. If the patient clearly expresses his/her refusal, the doll should be removed accordingly. Behaviors such as active approaching the doll, hugging/kissing the doll, and talking with the doll are considered the patient's acceptance of the doll. In general, the doll should not be directly removed from the patient. However, when the patient is too tired, the practitioner should temporarily remove the doll in a way that is acceptable to the patient $(2,12)$.

\section{Summary}

$\mathrm{AD}$ is an irreversible, progressive disease. The progression of $\mathrm{AD}$ is associated with a variety of physical and mental problems, such as cognitive decline, language disorder, emotional instability, and inability of self-care, levying considerable burdens and challenges to society, families, and the health care system (1). As an emerging and safe nonpharmacological treatment, DT offers a simple and cost-effective treatment option for AD. It can effectively improve the cognitive function and memory of $\mathrm{AD}$ patients, reduce agitated behavior, improve mood, and enhance quality of life (8). However, the clinical efficacy of DT has not yet been adequately investigated in high-quality studies, and there are certain ethical controversies in the implementation of DT. Therefore, whether DT can be widely applied for treating AD in clinical settings needs to be further explored $(9,16,20-22)$. In summary, DT is a feasible and effective intervention for $\mathrm{AD}$, with few adverse effects.

\section{Acknowledgments}

Funding: Nursing project of Nanjing Drum Tower Hospital: ZSA864.

\section{Footnote}

Reporting Checklist: The authors have completed the Narrative Review reporting checklist. Available at http:// dx.doi.org/10.21037/apm-21-853

Conflicts of Interest: All authors have completed the ICMJE uniform disclosure form (available at http://dx.doi. org/10.21037/apm-21-853). The authors have no conflicts of interest to declare.

Ethical Statement: The authors are accountable for all aspects of the work in ensuring that questions related to the accuracy or integrity of any part of the work are appropriately investigated and resolved.

Open Access Statement: This is an Open Access article distributed in accordance with the Creative Commons Attribution-NonCommercial-NoDerivs 4.0 International License (CC BY-NC-ND 4.0), which permits the noncommercial replication and distribution of the article with the strict proviso that no changes or edits are made and the original work is properly cited (including links to both the formal publication through the relevant DOI and the license). See: https://creativecommons.org/licenses/by-nc-nd/4.0/. 


\section{References}

1. Williamson HJ, McCarthy MJ, Garcia YE, et al. Addressing the Needs of Rural Caregivers of Individuals With Alzheimer's Disease and Related Dementias During and Beyond Coronavirus Disease 2019 (COVID-19). Public Policy Aging Rep 2020;30:178-80.

2. Mounsey AL, Zeitler MR. Cerebrospinal Fluid Biomarkers for Detection of Alzheimer Disease in Patients with Mild Cognitive Impairment. Am Fam Physician 2018;97:714-5.

3. Mendiola-Precoma J, Berumen LC, Padilla K, et al. Therapies for Prevention and Treatment of Alzheimer's Disease. Biomed Res Int 2016;2016:2589276.

4. Logsdon RG, Gibbons LE, McCurry SM, et al. Assessing quality of life in older adults with cognitive impairment. Psychosomatic Medicine 2002;64:510-9.

5. Strøm BS, Ytrehus S, Grov EK. Sensory stimulation for persons with dementia: a review of the literature. J Clin Nurs 2016;25:1805-34.

6. Cummings J, Lee G, Ritter A, et al. Alzheimer's disease drug development pipeline: 2019. Alzheimers Dement (N Y) 2019;5:272-93.

7. Zhang T, Liu CJ, Ni ZL. Association of Access to Healthcare with Self-Assessed Health and Quality of Life among Old Adults with Chronic Disease in China: Urban Versus Rural Populations. International Journal of Environmental Research and Public Health 2019;16:2592.

8. Green L, Matos P, Murillo I, et al. Use of Dolls as a Therapeutic Intervention: Relationship to Previous Negative Behaviors and Pro Re Nata (prn) Haldol Use Among Geropsychiatric Inpatients. Archives of Psychiatric Nursing 2011;25:388-9.

9. McCready L. Doll Therapy: An Approach to Calm and Enhance the Well-Being of Individuals with Agitated Dementia. Nursing Research 2017;66:E17-E.

10. Bisiani L, Angus J. Doll therapy: a therapeutic means to meet past attachment needs and diminish behaviours of concern in a person living with dementia--a case study approach. Dementia (London) 2013;12:447-62.

Cite this article as: Cai X, Zhou L, Han P, Deng X, Zhu H, Fang F, Zhang Z. Narrative review: recent advances in doll therapy for Alzheimer's disease. Ann Palliat Med 2021;10(4):4878-4881. doi: 10.21037/apm-21-853
11. Balzotti A, Filograsso M, Altamura C, et al. Comparison of the efficacy of gesture-verbal treatment and doll therapy for managing neuropsychiatric symptoms in older patients with dementia. Int J Geriatr Psychiatry 2019;34:1308-15.

12. Pezzati R, Molteni V, Bani M, et al. Can Doll therapy preserve or promote attachment in people with cognitive, behavioral, and emotional problems? A pilot study in institutionalized patients with dementia. Front Psychol 2014;5:342.

13. Ehrenfeld M, Bergman R. The Therapeutic Use of Dolls. Perspectives in Psychiatric Care 1995;31:21-2.

14. Moyle W, Murfield J, Jones C, et al. Can lifelike baby dolls reduce symptoms of anxiety, agitation, or aggression for people with dementia in long-term care? Findings from a pilot randomised controlled trial. Aging \& Mental Health 2019;23:1442-50.

15. Hazan C, Shaver P. Romantic love conceptualized as an attachment process. J Pers Soc Psychol 1987;52:511-24.

16. Miesen, B.. Care-giving in dementia: contours of a curriculum. Dementia 2010;9:473-89.

17. Alander H, Prescott T, James IA. Older adults' views and experiences of doll therapy in residential care homes. Dementia (London) 2015;14:574-88.

18. Shin JH. Doll Therapy: An Intervention for Nursing Home Residents With Dementia. J Psychosoc Nurs Ment Health Serv 2015;53:13-8.

19. Burke SL, Cadet T, Alcide A, et al. Psychosocial risk factors and Alzheimer's disease: the associative effect of depression, sleep disturbance, and anxiety. Aging \& Mental Health 2018;22:1577-84.

20. Mitchell G, Templeton M. Ethical considerations of doll therapy for people with dementia. Nursing Ethics 2014;21:720-30.

21. de la Cuesta-Benjumea C. Strategies for the relief of burden in advanced dementia care-giving. J Adv Nurs 2011;67:1790-9.

22. Ng QX, Ho CYX, Koh SSH, et al. Doll therapy for dementia sufferers: A systematic review. Complementary Therapies in Clinical Practice 2017;26:42-6. 